\title{
Actividad física, tabaquismo y consumo de alcohol, en un grupo de estudiantes universitarios
}

\author{
Physical activity and tobacco and alcohol use in a group of \\ university students
}

\author{
Sonia C. Mantilla-Toloza ${ }^{1}$, Antonia Gómez-Conesa ${ }^{2}$ y \\ Maria D. Hidalgo-Montesinos ${ }^{3}$
}

\footnotetext{
1 Departamento de Fisioterapia, Universidad de Pamplona, Colombia. sonia.mantilla@unipamplona.edu.co 2 Departamento de Fisioterapia, Universidad de Murcia, España. agomez@um.es

3 Departamento de Psicología Básica y Metodología, Universidad de Murcia, España. mhidalg@um.es
}

Recibido 28 Enero 2011/Enviado para Modificación 9 Octubre 2011/ Aceptado 25 Octubre 2011

\section{RESUMEN}

Objetivo Determinar la prevalencia de actividad física, tabaquismo y consumo de alcohol, en un grupo de estudiantes universitarios.

Metodología Con una muestra de 548 estudiantes del área socio-sanitaria (431 mujeres y 117 hombres, edad media: 20 años DT: 2,3) se aplicó la versión abreviada del Cuestionario Internacional de Actividad Física y una encuesta sobre hábitos de vida, para obtener información relacionada con el consumo de alcohol y tabaquismo. Se calcularon el índice de actividad física, los niveles de actividad, porcentaje de tabaquismo y consumo de alcohol. Se aplicó la prueba $V$ de Cramer para establecer asociación entre variables, la prueba t para evaluar las diferencias de actividad física en función del género y la prueba Brown Forsythe en función de la titulación. Resultados El 80 \% de los estudiantes se clasificaron como regularmente activos. Se encontraron diferencias significativas entre Índice de Actividad Física, género $(p=0,0)$ y titulación $(p=0,038)$. La prevalencia de tabaquismo fue del $33 \%$ y de consumo de alcohol fue del $58 \%$; sin diferencias significativas entre género. No se encontraron asociaciones significativas entre actividad física, tabaquismo y consumo de alcohol.

Conclusiones Un porcentaje alto de estudiantes practican actividad física de forma regular. Sin embargo, una parte de ellos no alcanza el parámetro mínimo para obtener beneficios en la salud. El porcentaje de consumo de alcohol y tabaquismo fue elevado. Se requiere implementar programas de promoción de hábitos saludables en universitarios, específicamente hacia la optimización de la práctica deportiva, la prevención y disminución del tabaquismo y consumo de alcohol.

Palabras Clave: Actividad física; evaluación; tabaquismo; utilización de bebidas alcohólicas (fuente: DeCS, BIREME) 


\begin{abstract}
Objective Determining the prevalence of physical activity, smoking and drinking alcohol in a group of university students studying in the social-health sciences' area. Methodology A shortened version of the International Physical Activity Questionnaire was used with a sample of 548 students from the social-health sciences area (431 females and 117 males, average 20 years old: 2.3 SD) and a survey about living habits for obtaining information related to drinking alcohol and smoking. The physical activity index, activity levels, percentage of smoking and drinking alcohol were all calculated. The Cramer's V coefficient was used for establishing an association between variables, the t-test for evaluating differences in physical activity regarding gender and the Brown Forsythe test concerning qualifications.

Results $80 \%$ of the students were classified as being regularly active. Significant differences were found between the Physical Activity Index, gender $(p=0.0)$ and qualifications $(p=0.038)$. Smoking prevalence was $33 \%$ and drinking alcohol $58 \%$, no significant differences being found regarding gender. No significant associations were found between physical activity, smoking and drinking alcohol.

Conclusions A high percentage of students regularly took part in physical activity. However, some of them did not reach the minimum parameters for obtaining healthrelated benefits. The percentage of students consuming alcohol and smoking was high. Programs must be implemented for promoting healthy habits in university students, specifically aimed at optimizing taking part in sports and preventing and reducing smoking and drinking alcohol.
\end{abstract}

Key Words: Physical activity, evaluation, smoking, drinking alcohol (source: $\mathrm{MeSH}$, $N L M)$.

os adultos jóvenes representan un grupo altamente vulnerable frente a la influencia de la sociedad actual en lo que respecta a la adopción estilos de vida por lo general caracterizados por conductas de riesgo para la salud, como por ejemplo dietas saturadas en grasa que conllevan a niveles elevados de colesterol, consumo de tabaco, alcoholismo y sedentarismo. La universidad es un lugar que facilita el contacto con este tipo de población, constituyéndose como un lugar estratégico para promover patrones de comportamiento que favorezcan la salud y caminos efectivos que conlleven al incremento de la participación en hábitos saludables.

Además, es bien reconocido que las enfermedades cardiovasculares representan la principal causa de morbi- mortalidad a nivel mundial $(1,2)$. Por eso resulta necesario indagar y actuar tempranamente en los hábitos de vida de los jóvenes, para prevenir y disminuir esas altas cifras. El primer paso en el diseño de acciones de promoción de hábitos saludables es el establecimiento de líneas de base que permitan caracterizar los comportamientos de salud en la población. En este trabajo se determinó el 
nivel de actividad física, consumo de alcohol y tabaquismo, de un grupo de estudiantes universitarios que en el futuro conformarán el gremio de profesionales del área socio-sanitaria; cuya labor involucrará la habilidad para suscitar la adopción y mantenimiento de hábitos saludables.

\section{MÉTODOS}

\section{Sujetos}

Este es un estudio descriptivo, de tipo transversal que se realizó con 548 estudiantes universitarios de las titulaciones de Fisioterapia, Enfermería, Medicina, Psicología y Magisterio, de la Universidad de Murcia, España. Dichas titulaciones fueron seleccionadas debido a la estrecha relación que tiene su perfil ocupacional con la promoción de hábitos saludables y calidad de vida. Como criterios de inclusión se establecieron pertenecer al primer año académico de cualquiera de las anteriores titulaciones y tener menos de 30 años de edad. De esta manera, la muestra estuvo constituida por 431 mujeres (79\%) y 117 hombres (21\%), con una edad media de 20 años (DT: $2,3)$.

Instrumentos

Para la recogida de la información sobre actividad física se utilizó la versión abreviada del Cuestionario Internacional de Actividad Física IPAQ, que se aplicó colectivamente. El IPAQ, es un instrumento creado por un grupo de expertos internacionales convocados por el Instituto Karolinska, la Universidad de Sydney, la OMS y el CDC, con el fin de unificar las dimensiones de evaluación y medición de la actividad física, para facilitar la comparación de resultados entre diferentes países. Estructurado en 7 items, el IPAQ corto permite registrar los valores de actividad física semanal en tiempo total y consumo calórico, a partir del cálculo del Indice de Actividad física (IAF) cuyo valor corresponde al producto de la intensidad (en METs), por la frecuencia (semanal), por la duración (minutos) de la actividad. Después de obtener el IAF, los sujetos se clasifican en uno de los 3 niveles de actividad física (NAF), de acuerdo a los siguientes criterios: Bajo: No hay registro de actividad física o si, pero sin alcanzar los niveles medio y alto; Medio: Alcanzan un registro entre 600 y 1499 MET-min/semana; y, Alto: Obtienen un IAF mayor o igual a 1500 METs-min-semana. El IPAQ ha sido validado en diversos estudios $(3,4)$.

La información sobre el tabaquismo y consumo de alcohol se obtuvo a partir de la aplicación de una encuesta sobre hábitos de vida desarrollada por 
el grupo de Investigación "Fisioterapia y Promoción de la Salud" del Departamento de Fisioterapia de la Universidad de Murcia; con el fin de facilitar la obtención de información relacionada tanto de salud general, como de las actividades cotidianas que constituyen los hábitos de vida de la población de estudio. Los ítems relacionados con tabaquismo y consumo de alcohol que se utilizaron en esta encuesta, han sido aplicados en investigaciones previas (5-7) para determinar la frecuencia de consumo.

\section{Procedimiento}

La recogida de la información se realizó entre los meses de marzo y junio, correspondientes al segundo cuatrimestre académico de 2008, bajo la supervisión y adopción de las normas éticas de la Universidad de Murcia. La pesquisa fue obtenida a través de la aplicación de una encuesta autodiligenciada, en una fecha establecida para cada grupo, en cada titulación y siguiendo un protocolo que incluyó los siguientes pasos:

- Presentación de los investigadores, del proyecto de investigación y sus objetivos,

- Mención de la importancia de la participación de los estudiantes en la Investigación, de la veracidad de la información y confidencialidad de los datos obtenidos,

- Explicación detallada acerca de la forma de contestar cada cuestionario, solicitando que se respondiera de acuerdo al criterio personal,

- Solicitud del consentimiento para participar en el estudio, puesto que la participación fue voluntaria,

- Distribución, diligenciamiento y recogida del material.

Durante la cumplimentación se aclararon dudas y se retroalimentó la importancia de participar y de la veracidad de los datos.

Análisis de la información

Los datos fueron consignados originalmente en un formato de ingreso de datos, de acuerdo con las variables del estudio y la fuente de la información. Para el análisis se utilizó el programa SPSS versión 14. Después de calcular el IAF, se realizó la distribución de frecuencias respecto a los NAF, tabaquismo y consumo de alcohol. Se aplicó la prueba $\mathrm{V}$ de Cramer para establecer asociación entre variables. Además, se aplicó la prueba t corregida para evaluar las diferencias en actividad física en función del género, la prueba Brown Forsythe en función de la titulación, seguida de la prueba de Games Howell para establecer comparaciones entre parejas de medias del IAF por titulación. 


\section{RESULTADOS}

La Tabla 1 muestra las características descriptivas de la muestra en estudio. La media del IAF fue de 2523 mets/min/semana (D.T: 2 982,4). Al distribuir la muestra por género, el IAF fue mayor para los hombres (3 $656 \mathrm{mets} / \mathrm{min} /$ semana DT: 3 593,8) que para las mujeres (2 $215 \mathrm{mets} / \mathrm{min} / \mathrm{semana}$ DT: 2 $717,8)$. Esta diferencia fue significativa entre ambos grupos $(p=0,000)$. También se encontraron diferencias en el IAF entre titulación, con el valor más alto en la titulación de Fisioterapia (2 943 mets/min/semana DT: 2 962,2) y el más bajo en psicología (2 $027 \mathrm{mets} / \mathrm{min} / \mathrm{semana}$ DT: 2 151,2). Estas diferencias fueron significativas $(\mathrm{p}=0,038)$. Después de aplicar pruebas posthoc para establecer comparaciones entre parejas de medias, sólo se encontraron diferencias significativas entre la titulación de Fisioterapia y Psicología $(\mathrm{p}=0,021)$ (Tabla 2).

Tabla 1. Características descriptivas de los sujetos de estudio

\begin{tabular}{|c|c|c|c|}
\hline Característica & $\begin{array}{l}\text { Todos } \\
(n=548)\end{array}$ & $\begin{array}{l}\text { Hombres } \\
(\mathrm{n}=117)\end{array}$ & $\begin{array}{r}\text { Mujeres } \\
(n=431)\end{array}$ \\
\hline Edad (años) & $20( \pm 2,32)$ & $20,45( \pm 2,69)$ & $19,87( \pm 2,19)$ \\
\hline Titulación & n (\%) & $\mathrm{n}(\%)$ & $\mathrm{n}(\%)$ \\
\hline Fisioterapia & $147(27)$ & $46(39)$ & $101(23)$ \\
\hline Psicología & $154(28)$ & $24(21)$ & $130(30)$ \\
\hline Enfermería & $138(25)$ & $19(16)$ & $119(28)$ \\
\hline Educación & $53(9)$ & $7(6)$ & $46(11)^{\prime}$ \\
\hline Medicina & $56(11)$ & $21(18)$ & $35(8)$ \\
\hline Consumo de tabaco & & \multicolumn{2}{|c|}{ No fuma } \\
\hline Nunca ha fumado & $319(58)$ & & \\
\hline $\begin{array}{l}\text { Actualmente no, pero ha sido } \\
\text { fumador }\end{array}$ & $47(9)$ & $54(46)$ & $175(41)$ \\
\hline Algunas veces & $62(11)$ & \multicolumn{2}{|c|}{ Si fuma } \\
\hline $\begin{array}{l}\text { Sólo los fines de semana } \\
\text { Menos de } 10 \text { diarios } \\
\text { Entre } 10 \text { y } 20 \text { diarios }\end{array}$ & $\begin{array}{c}17(3) \\
58(11) \\
45(8)\end{array}$ & $63(54)$ & $256(59)$ \\
\hline $\begin{array}{l}\text { Consumo de alcohol } \\
\text { Nunca o casi nunca }\end{array}$ & $231(42)$ & \multicolumn{2}{|c|}{ No consume } \\
\hline $\begin{array}{c}\text { Sólo los fines de semana } \\
\text { Diariamente }\end{array}$ & $\begin{array}{c}307(56) \\
10(2)\end{array}$ & Si co & $245(57)$ \\
\hline $\begin{array}{l}\mu \text { Indice de Actividad Física } \\
\text { IAF (met/min } / \mathrm{sem})^{a}\end{array}$ & $\begin{array}{c}2523 \\
( \pm 2982,4)\end{array}$ & $3656( \pm 3593,8)$ & $\begin{array}{c}2215 \\
( \pm 2717,8)\end{array}$ \\
\hline \multicolumn{4}{|l|}{ Nivel de Actividad Física NAF ${ }^{\mathrm{b}}$} \\
\hline $\begin{array}{l}\text { Bajo } \\
\text { Medio } \\
\text { Alto }\end{array}$ & $\begin{array}{l}110(20) \\
155(28) \\
283(52)\end{array}$ & $\begin{array}{l}14(12) \\
23(20) \\
80(68)\end{array}$ & $\begin{array}{l}96(22) \\
132(31) \\
203(47)\end{array}$ \\
\hline $\begin{array}{l}\text { Cumplir con la recomendación } \\
\text { de actividad física }{ }^{c}\end{array}$ & $438(80)$ & $103(87)$ & 335 (78) \\
\hline
\end{tabular}


Respecto a la distribución de la muestra de acuerdo al NAF, 110 estudiantes $(20 \%)$ se clasificaron en el nivel bajo, 155 estudiantes ( $28 \%)$ se clasificaron en el nivel medio y en el nivel alto se agruparon 283 estudiantes (52\%). Teniendo en cuenta que la recomendación mínima de actividad física con la que empiezan a obtenerse beneficios en la salud se registra a partir de la participación en por lo menos 30 minutos de actividad física moderada durante 5 días (2), o su equivalente en mets/min/semana, se considera que el $80 \%$ de la muestra cumple con esta condición (hombres $87 \%$, mujeres $78 \%$ ). Aunque se encontraron diferencias estadísticamente significativas en la distribución de frecuencias del NAF en función de la variable género, el tamaño del efecto fue bajo (V-Cramer $=0,175 ; p=0,00$ ). En cuanto a la distribución de los NAF por titulación, Fisioterapia y Enfermería fueron las titulaciones que obtuvieron mejor porcentaje de actividad física regular ( $84 \%$ y $81 \%$, respectivamente); seguidas de Medicina (78 \%) Educación (77\%) y Psicología $(77 \%)$. No se encontraron correlaciones significativas entre NAF y titulación.

Tabla 2. Distribución del IAF, actividad física suficiente, tabaquismo y consumo de alcohol por titulación

\begin{tabular}{|c|c|c|c|c|}
\hline Titulación & $\begin{array}{c}\text { IAF } \\
(\mathrm{mets} / \mathrm{min} / \mathrm{sem})^{\mathrm{a}, \mathrm{b}} \\
\mu(\mathrm{DT})\end{array}$ & $\begin{array}{l}\text { Cumplir con la } \\
\text { recomendación } \\
\text { de actividad } \\
\text { física }^{\mathrm{e}}(\%)\end{array}$ & $\begin{array}{c}\text { Tabaquismo }^{c} \\
(\%)\end{array}$ & $\begin{array}{c}\text { Consumo } \\
\text { de alcohol }^{\mathrm{d}} \\
(\%)\end{array}$ \\
\hline $\begin{array}{l}\text { Fisioterapia } \\
\qquad(n=147)\end{array}$ & $2943( \pm 2962,2)$ & $124(84 \%)$ & $95(65 \%)$ & $88(60 \%)$ \\
\hline $\begin{array}{l}\text { Psicología } \\
(n=154)\end{array}$ & $2027( \pm 2151,2)$ & $118(77 \%)$ & $76(49 \%)$ & $103(67 \%)$ \\
\hline $\begin{array}{c}\text { Enfermería } \\
\qquad(n=138)\end{array}$ & $2838( \pm 3839,7)$ & $111(80 \%)$ & $75(54 \%)$ & $70(51 \%)$ \\
\hline $\begin{array}{l}\text { Educación } \\
\qquad(n=53)\end{array}$ & $2272( \pm 2988,9)$ & $41(77 \%)$ & $29(55 \%)$ & $29(55 \%)$ \\
\hline $\begin{array}{l}\text { Medicina } \\
(n=52)\end{array}$ & $2247( \pm 2148,3)$ & $44(78 \%)$ & $44(79 \%)$ & $27(48 \%)$ \\
\hline
\end{tabular}

$\overline{a, b, c, d}$ Diferencias estadísticamente significativas ( $p<0,05$, IC 95\%). Estas diferencias fueron analizadas con la prueba Brown Forsythea, Games Howellb y V-Cramerc, ${ }^{d}$.

eparticipar en al menos 30 minutos de actividad física moderada, cinco veces por semana ${ }^{2}$.

$\mathrm{Al}$ revisar el consumo de tabaco, se encontró que el $58 \%$ de los estudiantes nunca ha fumado, el $9 \%$ alguna vez lo hizo y el $33 \%$ actualmente son fumadores. Para el análisis del consumo según el género y la titulación, se conformaron dos grupos: fumadores, en el cual se incluyeron las categorías algunas veces, sólo los fines de semana, menos de 10 diarios, entre 10 y 20 diarios; y no fumadores en el que se incluyeron aquellos que nunca han fumado y los que actualmente no, pero han sido fumadores. De esta manera, los hombres resultan ser más fumadores (46\%) que las mujeres (41\%) pero estas diferencias no son estadísticamente significativas $(\mathrm{p}=0,280)$. Por titulación, son los estudiantes de Medicina los que 
más fuman (79 \%), seguidos de Educación (55 \%) y Enfermería (54 \%). Los menores porcentajes corresponden a Psicología (35\%) y Fisioterapia (35\%). Aunque se encontraron diferencias estadísticamente significativas, el tamaño del efecto fue bajo (V-Cramer $=0,182 ; \mathrm{p}=0,001$ ). Acerca de la relación entre actividad fisica y tabaquismo, no se encontraron asociaciones significativas $(\mathrm{p}=0,558)$.

En cuanto al consumo de alcohol, el $42 \%$ de los estudiantes nunca o casi nunca ha consumido bebidas alcohólicas, el $56 \%$ lo hace sólo los fines de semana y el $2 \%$ beben diariamente. Al distribuir por género, los hombres (62\%) consumen más alcohol que las mujeres (57\%), pero estas diferencias no son significativas $(\mathrm{p}=0,362)$. Por titulación, son los estudiantes de Psicología quienes más consumen, seguidos de Fisioterapia y Educación. Las titulaciones donde hay menos consumo son Enfermería y Medicina. Estas diferencias aunque significativas, tuvieron un tamaño del efecto bajo ( $\mathrm{V}-\mathrm{Cramer}=0,139 ; \mathrm{p}=0,031)$. Al igual sucedió con la asociación entre el NAF y el consumo de alcohol (V-Cramer= 0,106; $\mathrm{p}=0,047$ ).

La Tabla 2 muestra la media del IAF, el porcentaje de sujetos que fuman, consumen alcohol y también los que cumplen con la recomendación mínima de actividad física; distribuidos de acuerdo a la titulación.

\section{DISCUSIÓN}

Los comportamientos de salud establecidos durante la adolescencia y adultez temprana pueden tener un impacto significativo en la adultez tardía. Como parte de este grupo poblacional, los estudiantes universitarios están mas fácilmente expuestos a comportamientos que representan riesgos y son candidatos prioritarios para los programas de prevención y promoción de la salud. En este estudio se ha determinado el comportamiento de la actividad física, tabaquismo y consumo de alcohol en una muestra de ellos.

Tabla 3. Prevalencia de actividad física suficiente para alcanzar beneficios en la salud, en algunos estudios realizados en población universitaria

\begin{tabular}{ccc}
\hline Referencia & $\mathrm{n}$ & $\begin{array}{c}\text { Porcentaje de actividad } \\
\text { física suficiente }\end{array}$ \\
\hline Steptoe, $2001^{8}$ & 4170 & 65 \\
${\text { Steptoe, } 2002^{7}}^{9}$ & 207776 & 70 \\
${\text { Haase, } 2004^{9}}^{\text {Bothmer, } 2005^{6}}$ & 4229 & 62 \\
Burke,2005 & 328 & 71 \\
${\text { Lee, } 2005^{11}}^{10}$ & 594 & 43 \\
Estudio 2009 & 247 & 49 \\
\hline
\end{tabular}


Se ha encontrado que el $80 \%$ de los estudiantes universitarios son regularmente activos y que de éste porcentaje, el $52 \%$ tiene un nivel alto de actividad física. Esta es una cifra satisfactoria, si se tiene en cuenta el aumento progresivo de la vulnerabilidad a factores de riesgo de enfermedades crónicas no transmisibles, entre ellos el sedentarismo. Pocas investigaciones han encontrado valores positivos al evaluar la actividad física de los jóvenes universitarios (6-11) (tabla 3), como también lo registró Irwin (12) a través de una revisión sistemática, en la que comparó la prevalencia de actividad física ejecutada en niveles adecuados para obtener beneficios en la salud, en 19 estudios realizados con estudiantes universitarios de 27 países diferentes. Como patrón de referencia para medir la cantidad de actividad física adecuada usó la recomendación del Centro para el Control y la Prevención de Enfermedades (CDC) y el Colegio Americano de Medicina Deportiva (ACSM) de acumular 30 minutos o más de actividad física de intensidad moderada, la mayoría o preferiblemente todos los días de la semana. Con respecto a esta guía, más de la mitad de estudiantes universitarios en Estados Unidos y Canadá no fueron suficientemente activos para obtener beneficios en la salud. Algo similar ocurrió en el resto de países, aunque los estudiantes australianos parecieron tener los más altos niveles de actividad física suficiente. En el presente trabajo se obtuvo un mejor porcentaje de actividad física respecto a los estudios previos, pero difiere metodológicamente respecto a los instrumentos utilizados, los parámetros de evaluación y la metodología. Probablemente los mejores resultados se deriven a partir de la selección de la muestra, la cual estuvo conformada por estudiantes de titulaciones relacionadas con la salud, en donde directa o indirectamente se adquieren actitudes, conocimientos, prácticas saludables y conciencia de las ventajas que tiene su adopción; por lo menos en mayor medida que en otro tipo de titulación.

Esta apreciación podría sustentarse con el modelo que han propuesto Sallis y Hovell (13), quienes consideran que el comportamiento del individuo respecto a la actividad física está determinado por la interacción de las condiciones ambientales, sociales, cognitivas, fisiológicas y personales. Condiciones que seguramente son inherentes en el ambiente universitario de las facultades de salud.

Otra razón por la que puede explicarse el alto grado de actividad física encontrado es que el nivel educativo puede incidir en la adopción de hábitos saludables, como se ha registrado en estudios previos $(14,15)$ en los que se ha determinado que poseer un mayor nivel educativo tiene un efecto positivo sobre la salud; a pesar de que el hecho de ingresar a la universidad coincide con una etapa que puede acompañarse de prácticas que podrían representar riesgos (por 
ejemplo sexo inseguro, tabaquismo) $(7,16)$ que impiden una mejor salud en el promedio de individuos jóvenes.

Aunque la prevalencia de actividad fue alta y la media en el ÍAF (2 523 mets/ $\mathrm{min} / \mathrm{sem}$ DT 2982,4) indique que en promedio se cumple con la cantidad mínima de actividad fisica recomendada para obtener beneficios en la salud, el $20 \%$ de la muestra estudiada tuvo un nivel insuficiente de actividad. De esta manera podría considerarse la planificación de estrategias curriculares o extracurriculares, que promuevan la vinculación de este grupo de individuos en la práctica de actividad física y a su vez el mantenimiento para aquellos que cumplen con la recomendación mínima.

En este trabajo se encontraron diferencias estadísticamente significativas en el IAF de acuerdo con la variable género. Este es un patrón constante, ya que la actividad física es uno de los pocos comportamientos relacionados con la salud típicamente más prevalentes en hombres que en mujeres $(17,18)$. Se ha establecido que el origen de estas diferencias se debe a la preferencia por ciertas actividades (19), a la diferencia de roles entre género (20) y a la influencia de factores sociales y culturales que determinan esos roles $(16,21)$.

Las titulaciones de Fisioterapia y Enfermería fueron las que registraron mejores valores de actividad física, pero sólo se encontraron diferencias significativas en el IAF entre estudiantes de Fisioterapia y Psicología. Probablemente porque los estudiantes de Fisioterapia tienen una relación directa con la concepción del movimiento, la importancia y ejecución de actividad física desde el rol de su formación y esto no sucede con los estudiantes de Psicología. Sin embargo el promedio del IAF en ambos casos sobrepasa los niveles recomendados de actividad.

En esta muestra, el $42 \%$ de los estudiantes son fumadores. La proporción de mujeres fumadoras (41\%) fue menor que la de los hombres (46\%), pero estas diferencias no fueron significativas. Estos datos son similares a los registrados por Steptoe (7), quienes después de comparar el comportamiento de éste hábito en 23 países, encontraron las más altas tasas de tabaquismo en Grecia, Italia, Portugal y España (Para éste último, los valores fueron $46 \%$ para las mujeres y $36 \%$ para los hombres). Las cifras del presente estudio son algo preocupantes, teniendo en cuenta que son mayores que las registradas por el Ministerio de Sanidad y Consumo de España, para el total de población adulta española (22), que en el año 2003 halló un porcentaje de tabaquismo de $38 \%$ para los hombres y de $25 \%$ para las mujeres. 
Respecto al consumo de alcohol, el $58 \%$ de los universitarios consume bebidas alcohólicas, sin diferencias significativas entre hombres y mujeres. Esta cifra es mayor que la encontrada por otros autores $(6,7)$ quienes además registraron mayor consumo de alcohol en los hombres que en las mujeres.

Los resultados de la distribución del tabaquismo y consumo de alcohol según la titulación muestran que en psicología y fisioterapia es donde hay mayor consumo de alcohol y menos fumadores, mientras que medicina es la titulación con mayor número de fumadores y menor cantidad de consumidores de bebidas alcohólicas. Teniendo en cuenta que la prevalencia de consumo de alcohol y tabaquismo es relativamente alta, existe una contraposición entre éstos comportamientos y los niveles adecuados de actividad física mencionados.

Los hallazgos de este estudio permiten discernir en aspectos relacionados con la salud de los jóvenes en la universidad, que conllevan a la construcción de la salud en la comunidad. En general se ha encontrado un nivel de actividad fisica adecuado, pero también altos porcentajes de tabaquismo y consumo de alcohol, situaciones que deberían estudiarse con mayor profundidad, más aún teniendo en cuenta que los resultados provienen de sujetos que cursan titulaciones relacionadas con el área sociosanitaria, que de alguna manera contribuirán al desarrollo de hábitos de vida en el futuro. El campus universitario es un escenario ideal para involucrar a un amplio número de jóvenes adultos en planes de educación y promoción de la salud, con ayuda de los educadores; quienes deberían trabajar en la búsqueda, adopción y sobre todo, el mantenimiento de prácticas protectoras para reducir comportamientos de riesgo a través de la academia y la educación integral.

Este fue un estudio descriptivo y por lo tanto no pueden establecerse inferencias causales a partir de los datos obtenidos. Aunque el hecho de que la muestra estuviera constituida sólo por estudiantes universitarios representa una fortaleza del estudio porque parcialmente se ha podido controlar el factor demográfico, también es una limitación puesto que los resultados no pueden considerarse como representativos de la población general de estudiantes universitarios a nivel local, regional o nacional. La información se ha obtenido a través de cuestionarios auto cumplimentados, por lo que su validez podría estar condicionada de acuerdo a la actitud y honestidad de los sujetos

\section{REFERENCIAS}

1. Jacobi E, Bull F, Neiman A. Cambios acelerados del estilo de vida obligan a fomentar la Actividad Física como prioridad en la región de las Américas. Revista Panamericana de Salud Pública 2003; 14:223-225. 
2. Center of Disease Control CDC. Physical activity and health: a report of the Surgeon General. Atlanta 1996.

3. Craig C, Marshall A, Sjostrom M, Bauman AE, Booth ML, Pratt M et al. International Physical Activity Questionnaire: 12- country Reliability and Validity. Med Sci Sports Exercise. 2003; 35:1381-95.

4. Brown WJ, Trost SG, Bauman A, Mummery K, Owen N. Test- retest reliability of four physical activity measures used in population surveys. J Sci Med Sport. 2004; 7:205-15.

5. Steptoe A, Wardle J, Cui W, Baban A, Glass K, Pelzer K. An international comparison of tobacco smoking, beliefs and risk awareness in university students from 23 countries. Addiction 2002; 97:1561-1571.

6. Bathmer M, Fridlund B. Gender differences in health habits and in motivation for a healthy lifestyle among Swedish university students. Nursing and Health Sciences 2005; 7:107118.

7. Steptoe A, Phil D, Cui W, Bellisle F, Zotti A, Baranyai R. Trends in smoking, diet, physical exercise and attitudes toward health in european university students from 13 countries, 19902000. Preventive Medicine 2002; 35:97-104.

8. Mantilla S, Gómez A, Hidalgo M. Prevalencia de actividad física en estudiantes de fisioterapia de la Universidad de Murcia. Fisioterapia 2008; 30:164-167.

9. Haase A, Steptoe A, Phil D, Sallis J, Wardle J. Leisure time physical activity in university students from 23 countries: associations with health beliefs, risk awareness and national economic development. Preventive Medicine 2004; 39:182-190.

10. Burke S, Carron A, Eys M. Physical activity context and university student's propensity to meet the guidelines Centers of Disease Control and Prevention/American College of Sports Medicine 2005; 11:171-176.

11. Lee R, Yuen A. Health-promoting behaviors and psychosocial well-being of university students in Hong Kong. Public Health Nursing 2005; 22(3):209-220.

12. Irwin J. Prevalence of university student's sufficient physical activity: a systematic review. Perceptual and Motor Skills 2004; 98:927-943.

13. Sallis JF, Hovell MF. Determinants of exercise behaviour. Journal of Exercise and Sports Science 1990; 11:307-330.

14. Grossman M. The demand for health, 30 years later: a very personal retrospective and prospective reflection. Health Economics 2004; 23:629-636.

15. Mantilla S. Actividad física en habitantes de 15 a 49 años de una localidad de Bogotá, Colombia, 2004. Rev. Salud pública 2008; 8 (Sup. 2): 69-80, 2006.

16. Häkkinen U, Järvelin M, Rosenquist G, Laitinen J. Health, schooling and lifestyle among young adults in Finland. Health Economics 2006; 15:1201-1216.

17. Steptoe A, Wardle J, Fuller R, Holte A, Justo J, Sanderman R. Leisure-time physical exercise: prevalence, attitudinal correlatos, and behavioral correctas among young europeans from 21 countries. Preventive Medicine 1997; 26:845-854.

18. Gómez L, Duperly J, Lucumí D, Gómez R, Venegas A. Nivel de actividad física global en la población adulta de Bogotá (Colombia). Prevalencia y factores asociados. Gaceta Sanitaria 2005; 19(3):206-13.

19. Buckworth J, Nigg C. Physical activity, exercise and sedentary behaviour in college students. Journal of American College Health 2004; 53:28-34.

20. Cabrera G, Gomez L, Mateus J. Leisure time physical activity among women in a neighbourhood in Bogotá, Colombia: prevalence and socio- demographic correlates. Revista de Salud Pública (Bogotá) 2004; 20:1103-1109.

21. Denton M, Prus S, Walters V. Gender differences in health: a canadian study of the psychosocial, structural and behavioural determinants of health. Social Science and Medicine 2004; 58:2585-2600.

22. La Salud de la Población Española. Indicadores de Salud. Ministerio de Sanidad y Consumo. Secretaría General de Sanidad. Instituto de formación Sanitaria. 18 de abril de 2006. 